\title{
Las competencias del personal de consejería en rehabilitación para trabajar con poblaciones lesbianas, gays y bisexuales (LGB)
}

\author{
Competencies of the Rehabilitation Counselor to Work with Lesbian, Gay and Bisexual \\ Clients (LGB)
}

\section{Competências da equipe de orientação em reabilitação para trabalhar com populações lésbicas, gays e bissexuais (LGB)}

Raúl Rivera-Colón Universidad de Puerto Rico Recinto de Río Piedras San Juan, Puerto Rico raul.rivera7@upr.edu (D) https://orcid.org/0000-0003-2764-7798

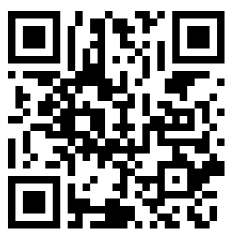

Maximino Ramos-Reyes 色 Universidad de Puerto Rico Recinto de Río Piedras San Juan, Puerto Rico maximino.ramos@upr.edu

Robinson Vázquez-Ramos Universidad de Puerto Rico Recinto de Río Piedras San Juan, Puerto Rico robinson.vazquez@upr.edu 
Resumen: El personal de consejería en rehabilitación debe proveer servicios a grupos diversos, incluidas personas lesbianas, gays y bisexuales (LGB). Este estudio cuantitativo de tipo descriptivo exploratorio auscultó las competencias de un grupo de consejería en rehabilitación para trabajar con la población LGB. La muestra estuvo constituida por 118 profesionales de esta consejería, a quienes se les reclutó por disponibilidad. Los instrumentos administrados fueron un cuestionario de datos sociodemográficos y la Escala de competencias del consejero en orientación sexual (SOCCS, por sus siglas en inglés) (Bidell, 2005). Se analizaron las variables con la prueba t y correlación de Spearman. El grupo participante mostró competencias moderadas en las subescalas del SOCCS (conocimiento, destrezas y actitudes) y en el promedio total de la escala. No se identificó un resultado estadísticamente significativo en cuanto a las variables de género y el escenario laboral del personal consejero. Se encontró una relación negativa estadísticamente significativa entre los años de experiencia en el ejercicio de la profesión y las subescalas de destrezas y actitudes. Estos resultados podrán ser utilizados para el desarrollo de programas de educación continua para profesionales de la consejería en rehabilitación con el propósito de mejorar el bienestar de sus clientes LGB.

Palabras claves: Competencias; personal consejero en rehabilitación; LGB.

Abstract: Rehabilitation counselors must provide services to diverse populations including lesbian, gay and bisexual (LGB) individuals. This quantitative study of descriptive exploratory type auscultated the competencies of a group of rehabilitation counselors to work with the LGB population. The sample consisted of 118 rehabilitation counseling professionals who were recruited utilizing a convenience sampling technique. The instruments administered were a sociodemographic data questionnaire and the Sexual Orientation Counselor Competence Scale (SOCCS), (Bidell, 2005). Variables were analyzed with the Spearman t-test and correlation. The participants showed moderate competences in the subscales of the SOCCS (knowledge, skills, and attitudes) and in the overall average of the Scale. No statistically significant result was identified in terms of gender variables and the work scenario of the counselors. A statistically significant negative relationship was found between years of working experience in rehabilitation counseling, the skills and attitudes subscales. These results may be used for the development of continuing education programs for rehabilitation counselors with the aim of improving the well-being of their LGB clients.

Keywords: Competencies, LGB, rehabilitation counseling.

Resumo: A equipe de orientação em reabilitação deve fornecer serviços a diversos grupos, incluindo pessoas lésbicas, gays e bissexuais (LGB). Este estudo quantitativo do tipo exploratório descritivo auscultou as competências de um grupo de pessoas orientadoras em reabilitação para trabalhar com a população LGB. A amostra foi constituída por 118 profissionais de orientação, que foram recrutados de acordo a sua disponibilidade. Os instrumentos administrados estavam compostos de um questionário de dados sociodemográficos e a Escala de Competências de Conselheiros sobre Orientação Sexual (SOCCS) (Bidell, 2005). As variáveis foram analisadas com o teste t de Spearman e correlação. O grupo participante demonstrou moderadas competências nas subescalas da SOCCS (conhecimentos, habilidades e atitudes) e na média total da escala. Nenhum resultado estatisticamente significativo foi identificado em termos de variáveis de gênero e do cenário de trabalho do pessoal de orientação. Foi encontrada uma relação negativa estatisticamente significativa entre os anos de experiência na prática da profissão e as subescalas de habilidades e atitudes. Esses resultados podem ser usados para o desenvolvimento de programas de educação permanente para profissionais de orientação em reabilitação, com o objetivo de melhorar o bem-estar de seus clientes LGB.

Palavras-chave: Competências; orientadores de reabilitação pessoal; LGB. 


\section{Introducción}

La consejería en rehabilitación es una profesión que evoluciona constantemente. Surge en Estados Unidos por mandato legislativo y remonta sus orígenes con la Ley Smith-Fess de 1920 (Ley Pública 66-236), la cual promueve el inicio del Programa de rehabilitación vocacional para civiles (Villafañe, Velázquez y Báez, 2013). Esta profesión es considerada una especialización dentro de la consejería y su objetivo es servir a las personas con diversidad funcional, o sin esta, para que logren metas personales, ocupacionales y de vida (Villafañe et al., 2013). En los últimos años, a medida que el campo de la consejería en rehabilitación ha crecido, ha surgido la necesidad de servir a poblaciones más diversas y emergentes.

Esto es reconocido por el Código de Ética de Profesionales de la Consejería en Rehabilitación de Puerto Rico, en el cual se requiere que las personas con licencia para ejercer la consejería en rehabilitación sean competentes para atender a clientes de diversos orígenes y que no discriminen "por motivos de edad, color, cultura, impedimento, etnia, género, raza, nacionalidad, religión, orientación sexual, estado civil, afiliación política o estatus socioeconómico" (Departamento de Salud, 2013, p. 14). Se espera que las personas que desempeñan la profesión de consejería en rehabilitación sean multiculturalmente competentes, habiendo desarrollado en su formación profesional el conocimiento, las actitudes y las destrezas para trabajar eficazmente con individuos en una variedad de grupos culturales, sociales y étnicos (Departamento de Salud, 2013).

Convertirse en profesional de la consejería multicultural competente es un proceso complejo en el que la persona que desempeña esta profesión debe tomar conciencia de sus valores y prejuicios culturales, la visión de mundo de su cliente y las estrategias de intervención culturalmente apropiadas (Arredondo et al., 1996).

La definición contemporánea del multiculturalismo considera características culturales de edad, clase social, género, espiritualidad, origen étnico, nacionalidad, familia, orientación sexual y discapacidad (Richardson y Jacob, 2002).

Profesionales de la consejería multiculturalmente competente deben prestar atención a las necesidades únicas de los diferentes grupos y a las diferencias dentro del grupo. Los conocimientos, las actitudes, la sensibilidad y las destrezas necesarias para la práctica competente de la consejería con los miembros de un grupo minoritario pueden diferir de los necesarios para el trabajo competente con otro (Kocarek y Pelling, 2003). Las personas que ejercen la consejería en rehabilitación deben contar con las herramientas necesarias para ofrecer servicios de consejería a clientes de diversos grupos emergentes, incluyendo la población de lesbianas, gays y bisexuales (LGB).

La obligación profesional de las personas que trabajan la consejería en rehabilitación, desde la perspectiva de la consejería multicultural, es capacitar a sus clientes que pertenecen a grupos oprimidos sobre sus derechos y apoyarlos en los esfuerzos por lograr justicia social (Green, McCollum y Hays, 2008). La población LGB es uno de esos grupos que sigue sufriendo 
doi: http://dx.doi.org/10.15359/ree.23-3.20

URL: http://www.una.ac.cr/educare

CORREO: educare@una.cr

discriminación en muchos niveles: las leyes gubernamentales no son igualmente representativas, experimentan la exclusión, la condena de ciertos grupos religiosos y la estigmatización a nivel personal. Todas estas variables crean un ambiente de opresión cultural para los individuos LGB.

Las personas con orientación sexual LGB son discriminadas como resultado del heterosexismo que exhibe la cultura occidental (Daniel, Roysircar, Abeles y Boyd, 2004). En un estudio realizado en los Estados Unidos con 73 individuos LGB, Mays y Cochran (2001) informaron que el 75\% afirmó haber experimentado discriminación como resultado de su orientación sexual. Esta incluyó el despido de un trabajo o interacciones negativas con otras personas debido a su orientación sexual. Gran parte de las respuestas de las encuestas reportaron que se les negó la vivienda, experimentaron ataques físicos y verbales y tuvieron problemas para obtener servicios sociales. La mayoría de participantes dijo que las prácticas discriminatorias habían resultado en desordenes psiquiátricos como la depresión y la ansiedad, así como en una menor calidad de vida. La investigación concluyó que ser miembro de un grupo estigmatizado, así como experimentar o tener experiencia de discriminación tiene un efecto negativo sobre la salud mental. Estos resultados son cónsonos con las expresiones de Meyer (2003), quien señaló que las personas LGB a menudo pueden experimentar discriminación que puede afectar negativamente su salud mental. Lo que a su vez puede llevarlas a necesitar servicios de consejería que deben ser brindados por profesionales multiculturalmente competentes.

Las personas lesbianas, gays y bisexuales también son más propensas a buscar servicios de salud mental en comparación con las heterosexuales (Cochran, Sullivan, y Mays, 2003), y quienes proveen salud mental frecuentemente reportan tener clientes LGB (Murphy, Rawlings y Howe, 2002). Sin embargo, parece que la satisfacción con estos servicios es menor entre las minorías sexuales, según se informa debido a la homofobia, al sesgo heterosexista y a la falta de comprensión de las experiencias por las que atraviesan estas personas (Palma y Stanley, 2002). Aunque la satisfacción con el tratamiento aumenta cuando clientes LGB perciben a sus consejeros o consejeras competentes y sensibles a sus preocupaciones (Burckell y Goldfried, 2006).

\section{Competencias para trabajar con los clientes LGB}

De acuerdo con Roe (2002), el término competencias se refiere a la habilidad que profesionales aprenden para desempeñar efectivamente una tarea, trabajo o función. La palabra competencia se utiliza porque implica tener la capacidad de funcionar eficazmente dentro del marco de patrones de comportamiento culturalmente incorporados y definidos por un grupo. El Código de Ética de la Junta Examinadora de los Profesionales de la Consejería en Rehabilitación de Puerto Rico (JECR-PR) describe las competencias de dos maneras diferentes. En primer lugar, considera las competencias como un valor de la profesión, que requiere que las personas que desempeñan la consejería en rehabilitación trabajen dentro de su área de competencia y se esfuercen por mejorar continuamente sus destrezas y aplicarlas a su práctica (Departamento

4

Raúl Rivera-Colón, Maximino Ramos-Reyes y Robinson Vázquez-Ramos

Los artículos de la Revista Electrónica Educare del Centro de Investigación y Docencia en Educación de la Universidad Nacional, Costa Rica, se comparten bajo términos de la Licencia Creative Commons: Reconocimiento, No Comercial, Sin Obra Derivada 3.0 Costa Rica. Las autorizaciones adicionales a las aquí delimitadas se pueden obtener en el correo: educare@una.cr 
de Salud, 2013). En segundo lugar, el Código de Ética de la JECR-PR establece el desarrollo de competencias como un estándar ético. Afirma que las personas que ejercen la consejería en rehabilitación deben ser conscientes de la función que tiene la diversidad multicultural en la relación de consejería y abordarla. Afrontar temas multiculturales y asuntos relacionados con el poder dentro de la relación de consejería es un paso crítico para ayudar a profesionales de la consejería en rehabilitación a ser capaces de reconocer y tratar preocupaciones similares con sus clientes. Dada la diversidad de clientes que se atienden por las personas que ejercen la consejería en rehabilitación y las desigualdades sociales a menudo enfrentadas por esa clientela, es imprescindible que estos grupos profesionales puedan trabajar eficazmente y reconocer el impacto de factores multiculturales en el proceso de evaluación, en el desarrollo de la relación de consejería y en el proceso de rehabilitación en general (Departamento de Salud, 2013).

En la profesión de consejería en rehabilitación, las competencias para intervenir con los clientes LGB se basan en el trabajo teórico de las competencias de consejería multicultural (Arredondo et al., 1996), que incluye el desarrollo de conocimientos, actitudes y destrezas para trabajar eficazmente con individuos en una variedad de grupos culturales, sociales y étnicos. Aunque el enfoque de las competencias de consejería multicultural abarca cuestiones raciales y étnicas, Bidell (2005) tomó prestado el marco y lo aplicó al conocimiento, las actitudes y las destrezas necesarias para brindar servicios de consejería a clientes LGB.

\section{Conocimientos}

Las personas que ejercen la consejería en rehabilitación deben obtener conocimientos sobre temáticas relacionadas con la población LGB, las situaciones que pueden enfrentar y su visión cultural. Es útil contar con una amplia gama de conocimiento relacionado con la población LGB para proveer servicios de consejería que resulten en facilitación de recursos (Israel, Ketz, Detrie, Burke y Shulman, 2003). Por ejemplo, para ayudar a clientes LGB a afrontar el proceso de revelar la orientación sexual y entender la complejidad de las experiencias con su desarrollo y orientación sexual, las personas que practican la consejería necesitan entender la historia de discriminación, opresión, prejuicio y heterosexismo de los Estados Unidos. Un sujeto consejero competente necesita saber acerca de los recursos de la comunidad y grupos de apoyo que pueden beneficiar a sus clientes LGB. Además, las personas que proveen servicios de consejería personas ofensoras y a víctimas de violencia doméstica deben tener amplio conocimiento sobre la complejidad de las situaciones implícitas, para que puedan reconocer si esas complejidades incluyen la orientación sexual. Este tipo de conocimiento puede venir de una variedad de fuentes, tales como los trabajos en los cursos, las relaciones personales con personas LGB, y los procesos formales de capacitación. Las investigaciones han indicado que el conocimiento por sí solo es insuficiente para aumentar las competencias de las personas que ejercen la consejería (Kocarek y Pelling, 2003); las actitudes y destrezas de los profesionales de la consejería son esenciales en el desarrollo de las competencias para trabajar con sus clientes LGB. 
doi: http://dx.doi.org/10.15359/ree.23-3.20

URL: http://www.una.ac.cr/educare

CORREO: educare@una.cr

\section{Actitudes}

La actitud de quienes practican la consejería hacia las personas LGB se refiere a adquirir una mayor percatación de sí, de sus propios valores, prejuicios y creencias hacia su cliente o grupos con los cuales su cliente se identifica (Arredondo et al., 1996; Bidell, 2005). La investigación relacionada con las actitudes hacia las personas LGB es abundante (Arredondo et al., 1996; Sue, Arredondo y McDavis, 1992; Israel et al., 2003; Satcher y Schumacker, 2009).

Una investigación desarrollada por Balkin, Schlosser y Levitt (2009) encontró que las personas consejeras que eran más rígidas y autoritarias en sus creencias religiosas tendían a tener más actitudes y comportamientos homofóbicos; por lo tanto, estos grupos profesionales necesitan oportunidades para explorar cómo sus valores religiosos pueden influir en su intervención con clientes LGB, particularmente en el área de la homofobia. Israel et al. (2003) argumentan que es poco probable que las personas que practican la consejería crean que es moralmente incorrecto ser miembro de un grupo étnico minoritario; sin embargo, en contraste, pueden creer que la homosexualidad es un pecado. Particularmente en algunas tradiciones religiosas protestantes, el dicho "Ama al pecador, odia el pecado" es una mentalidad que predomina en algunos sistemas de creencias cristianos hacia la comunidad gay. La familia de origen o contexto social en el que se desarrolla y vive una persona está a menudo relacionada con las creencias, valores y sesgos que desarrolla. Por lo que, además del conocimiento y la reflexión sobre las actitudes de sus clientes LGB, quienes aconsejan necesitan oportunidades para practicar y aumentar sus destrezas para intervenir efectivamente con esta población.

\section{Destrezas}

El desarrollo de las destrezas de consejería para el trabajo con clientes LGB es un elemento crítico para lograr las competencias. Las destrezas de consejería para clientes LGB se refiere a participar en una variedad de servicios de ayuda que comprenden la comunicación verbal y no verbal, el uso efectivo de las condiciones facilitadoras de la relación de consejería y las intervenciones culturalmente apropiadas, incluyendo, la evaluación y diagnóstico de clientes LGB (Arredondo et al., 1996; Bidell 2005). Sin embargo, se han desarrollado pocos estudios empíricos para identificar estrategias de capacitación que aumentan las destrezas para intervenir con clientes LGB. Las actividades de juego de roles han formado parte de la educación del personal consejero. Kocarek y Pelling (2003) abogan por el uso de los ejercicios de juego de rol con temática LGB para aumentar las destrezas en el trabajo con esta población. Necesitan oportunidades para participar en actividades que aumenten sus destrezas para la intervención con clientes LGB.

Las investigaciones sobre los conocimientos, las actitudes y las destrezas de personal de consejería para realizar intervenciones con la población LGB han identificado la religiosidad, la homofobia y el heterosexismo como factores que influyen negativamente en el desarrollo de las competencias (Balkin et al., 2009; Rainey y Trusty, 2007).

6

Raúl Rivera-Colón, Maximino Ramos-Reyes y Robinson Vázquez-Ramos

Los artículos de la Revista Electrónica Educare del Centro de Investigación y Docencia en Educación de la Universidad Nacional, Costa Rica, se comparten bajo términos de la Licencia Creative Commons: Reconocimiento, No Comercial, Sin Obra Derivada 3.0 Costa Rica. Las autorizaciones adicionales a las aquí delimitadas se pueden obtener en el correo: educare@una.cr 
Además, otros estudios han encontrado que la exposición a personas y relaciones LGB (Herek, 2002), y la capacitación formal en consejería para la población LGB a través de talleres y cursos de posgrado (Pearson, 2003) influyen positivamente en las competencias para trabajar con la población LGB.

Las competencias de consejería para clientes LGB han sido estudiadas con personal consejero de familia (Henke, Carlson y McGeorge, 2009), de escolares y de comunidades (Bidell, 2005) y estudiantes de posgrado en consejería (Bidell, 2012). Sin embargo, no se han desarrollado investigaciones sobre las competencias en rehabilitación para brindar servicios de consejería a clientes LGB.

Para lograr efectividad en las intervenciones con la población LGB, el personal consejeros en rehabilitación necesita conocimiento de la comunidad LGB, autoconocimiento de las actitudes personales hacia las personas de la comunidad LGB y aprender destrezas para ofrecer consejería a clientes LGB de manera competente. Por lo tanto, el objetivo de esta investigación fue auscultar la percepción de profesionales de la consejería en rehabilitación sobre sus competencias para trabajar con clientes LGB. Conocer las competencias para trabajar específicamente con personas LGB proporciona un marco desde el cual pueden evaluar sus propias conocimientos, actitudes y destrezas para, de esta forma, desarrollarlos, reforzarlos o corregirlos.

\section{Método}

\section{Diseño de investigación}

El diseño que se utilizó para este estudio fue de tipo descriptivo exploratorio. Responde al abordaje de una temática poco estudiada, en la que se ausculta la percepción de profesionales de la consejería en rehabilitación en torno a sus competencias para intervenir con la población LGB. Según Hernández, Fernández y Baptista (2010), este diseño se enfoca en la descripción de las características o funciones del problema en cuestión, y facilita una mayor profundidad y comprensión del fenómeno en estudio. El proceso de recolección y análisis de datos es cuantitativo, utiliza encuestas que fueron analizadas mediante el programa estadístico Statistical Package for the Social Sciences (SPSS, por sus siglas en inglés, versión 23.0).

\section{Participantes}

En este estudio participaron voluntariamente 118 profesionales de la consejería en rehabilitación con licencia para ejercer en Puerto Rico. Los criterios de inclusión fueron: (a) tener 21 años de edad o más, (b) estar activo o activa o que lo hayan estado en la práctica de la profesión, $y_{1}$ (c) poseer licencia vigente de la Junta Examinadora de Consejeros en Rehabilitación de Puerto Rico (JECR-PR). La muestra se obtuvo por disponibilidad y se les garantizó su voluntariedad, confidencialidad y el derecho a abandonar la investigación cuando lo consideraran necesario. 
doi: http://dx.doi.org/10.15359/ree.23-3.20

URL: http://www.una.ac.cr/educare

CORREO: educare@una.cr

\section{Instrumentos}

El grupo de profesionales de la consejería en rehabilitación que accedieron a participar completó los siguientes cuestionarios: (a) Planilla de datos sociodemográficos y (b) Escala de competencias del consejero en orientación sexual (SOCCS, por sus siglas en inglés; Bidell, 2005). La Planilla de datos sociodemográficos contenía 8 preguntas que tenían el propósito de recoger datos demográficos de cada participantes como: la edad, el género, el grado académico, los años de experiencia, entre otros.

Las competencias de profesionales de la consejería en rehabilitación para realizar intervenciones con sus clientes LGB fueron exploradas utilizando la Escala de competencias del consejero en orientación sexual (SOCCS, por sus siglas en inglés), desarrollada por Bidell (2005). EI SOCCS fue creado como un instrumento que se utiliza para determinar las competencias autopercibidas de profesionales de la salud mental para el trabajo con clientes LGB. Específicamente, el SOCCS evalúa los conocimientos, destrezas y actitudes de personal consejero respecto al trabajo con clientes LGB (Bidell, 2005). Por lo tanto, el SOCCS se compone de tres subescalas que evalúan cada una de estas tres áreas. La subescala de actitudes, compuesta por diez preguntas; la subescala de destrezas con once preguntas, y la subescala de conocimiento que contiene ocho preguntas. El SOCCS (Bidell, 2005) se compone de 29 premisas, las cuales son contestadas con una escala de intensidad que contiene una gradilla de números escalonados donde el número 1 el más bajo y el número 7 el más alto. Los números oscilan entre la siguiente tres designaciones: nada cierto (1), algo cierto (4) y totalmente cierto (7). A mayor puntuación obtenida, mayores niveles de competencia para el trabajo con la población LGB.

Los investigadores obtuvieron la autorización escrita del autor para proceder con la traducción, validación y adaptación del instrumento. La consistencia interna (alfa de Cronbach) de los 29 ítems del SOCCS fue de $a=0.79(n=118)$, lo que sugiere una alta confiabilidad. Entre las subescalas del SOCCS, la consistencia interna de la subescala de conocimiento fue de $a=0.71$, para ocho ítems. La consistencia interna para la subescala de las actitudes fue de $a=0.85$, para diez ítems. Finalmente, la consistencia interna para la subescala de destrezas fue de $a=0.86$, para once ítems. Estos coeficientes de confiabilidad son comparables al estudio de validación original de Bidell (2005), quien mostró que la versión original del SOCCS exhibió buenas propiedades psicométricas. El alfa de Cronbach para el SOCCS global fue de $a=0.90$, para la subescala de actitudes fue de $a=0.88$, para la subescala de destrezas $a=0.91$ y para la subescala de conocimiento $a=0.76$. Esto indica una cantidad moderadamente alta de consistencia interna.

\section{Procedimiento}

Para garantizar los derechos de cada participante se tomaron en consideración los estándares éticos de la Junta Examinadora de Consejeros en Rehabilitación de Puerto Rico. Esta investigación fue aprobada por el Comité Institucional para la Protección de los Seres Humanos 
en la Investigación (CIPSHI) de la Universidad de Puerto Rico, Recinto de Río Piedras. Además, el estudio se llevó a cabo con el auspicio del Decanato de Estudios Graduados e Investigación (DEGI) de la Universidad de Puerto Rico, Recinto de Río Piedras, a través de los Fondos Institucionales para la Investigación (FIPI).

Las personas interesadas en participar conocieron de la investigación a través del Colegio de los Profesionales de la Consejería en Rehabilitación de Puerto Rico, Inc. (CPCR-PR). Este organismo nos permitió compartir con profesionales de la consejería en rehabilitación la invitación a participar del estudio. Una vez que indicaron su interés en participar en el estudio, los investigadores se aseguraron de que reunieran los criterios de inclusión: a) tener 21 años de edad o más; b) ser personal consejero en rehabilitación que al presente (o en el pasado) haya estado activo en la prestación de servicios; c) poseer licencia vigente de la Junta Examinadora de Consejeros en Rehabilitación de Puerto Rico (JECR-PR), según lo estipula el Departamento de Salud y lo regulan con leyes del Estado Libre Asociado de Puerto Rico para ejercer la profesión de consejero en rehabilitación. Quienes cumplieron con los requisitos para participar de la investigación completaron la hoja de consentimiento informado brindando a los investigadores su autorización para el manejo de los datos. Una vez obtenido el consentimiento, se les entregó la hoja de datos sociodemográficos y la Escala de competencias del consejero en orientación sexual (SOCCS) (Bidell, 2005). Luego de haber completado la recolección de datos se procedió con el análisis estadístico utilizando el programa Statistical Package for the Social Sciences (SPSS, por sus siglas en inglés, versión 23.0).

\section{Resultados}

A partir de la planilla de datos sociodemográficos se obtuvo una variedad de información sobre la muestra con respecto a las características personales, la capacitación y las experiencias profesionales. De los 118 participantes, 92 (78\%) se identificaron con el género femenino y 25 (21.2\%) con el género masculino, mientras 1 (.8\%) participante omitió la pregunta. La edad osciló de los 25 a los 65 años, con una edad promedio de 41.92 años (SD = 9.554). En cuanto al grado académico más alto que ostentan, 107 (67.3\%) participantes informaron tener grado de maestría, mientras 8 (5\%) informaron ostentar grado doctoral y $3(2.5 \%)$ no reportaron la data. Referente a los años ejerciendo la profesión de consejería en rehabilitación, 34 (28.8\%) reportaron 1 a 5 años de experiencia; 25 (21.2\%) de 6 a 10; 13 (11.0\%) 11 a 15 ; 23 (19.5\%) 16 a 20; 11 (9.3\%) 21 a 25; 6 (5.1\%) 26 a 29 y 5 (4.2\%) 30 o más; 1 (.8\%) no reportó los años de experiencia. Respecto al escenario laboral en el que ejercen la profesión de consejería en rehabilitación, 77 (65\%) informó trabajar en el sector público; mientras 21 (18\%) en el sector privado y 20 (17\%) estar en desempleo o no contestaron la pregunta. 
doi: http://dx.doi.org/10.15359/ree.23-3.20

URL: http://www.una.ac.cr/educare

CORREO: educare@una.cr

Como se indicó anteriormente, los investigadores obtuvieron la autorización para proceder con la traducción, validación y adaptación de la Escala de competencias del consejero en orientación sexual (SOCCS, por sus siglas en inglés; Bidell, 2005). Después de proceder con el estudio para la traducción, validación y adaptación del SOCCS, se computó un coeficiente alpha de Cronbach de .79, lo cual indica una cantidad moderada alta de consistencia interna. Mientras el coeficiente alpha de Cronbach computado por subescala fue de 0.85 para la subescala de actitudes, 0.86 para la subescala de destrezas y 0.71 para la subescala de conocimientos. Los ítems fueron calificados tanto por subescalas como de forma total o agregada, indican que mientras más altas las puntuaciones mayores niveles de competencia en orientación sexual. Bidell (2005) señaló que los puntajes bajos (1.00 -2.00) representan una competencia baja; los puntajes medios (3.00-5.00), una competencia moderada, y los puntajes altos (6.00-7.00) una competencia alta. La media para la puntuación total o agregada de participantes fue $4.49(\mathrm{DE}=0.80)$, lo que indica que su percepción se encuentra dentro del rango medio de competencias para intervenir con la población LGB. En cuanto a los resultados por subescalas, el grupo de participantes obtuvo una puntuación media de $5.79(\mathrm{DE}=1.27)$ en la subescala de actitudes, $3.72(\mathrm{DE}=1.35)$ en la subescala de destrezas, y $3.97(D E=1.14)$ en la subescala de conocimientos. Esto revela que, en promedio, están dentro del rango de competencias moderado en las áreas de actitudes, destrezas y conocimientos. A continuación, se presenta la Tabla 1 en la cual se desglosan los resultados.

Tabla 1: Competencias de participantes para intervenir con clientes LGB

\begin{tabular}{lcc}
\hline Competencias SOCCS & $M$ & $D E$ \\
\hline Actitudes & 5.79 & 1.27 \\
Destrezas & 3.72 & 1.35 \\
Conocimientos & 3.97 & 1.14 \\
\hline Total & 4.49 & 0.80 \\
\hline
\end{tabular}

Nota: Elaboración propia.

Por otro lado, se realizó el análisis de Prueba $t$ para muestras independientes. Esta prueba paramétrica se efectuó para explorar si existía diferencia estadísticamente significativa entre las percepciones de los participantes con respecto a su nivel de competencia para trabajar con clientes LGB (promedio total, actitudes, destrezas y conocimientos en el SOCCS) y las variables de género y el escenario en el que laboran. En cuanto a la variable género, no se encontró diferencia estadísticamente significativa en la puntuación total de profesionales de la consejería en rehabilitación. Para la muestra de género masculino el resultado fue $(n=25, M=4.68, D E=$ $.78),(t(1.30)=115, p=.19)$ y en la muestra de género femenino fue $(n=92, M=4.45, D E=.80)$, $(t(1.30)=115, p=.19)$. En las subescalas de actitudes, destrezas y conocimientos tampoco se encontró diferencia estadísticamente significativa con respecto a la variable género. La Tabla 2 que se presenta a continuación detalla los datos por cada subescala. 
Tabla 2: Diferencia en las competencias de participantes en función del género

\begin{tabular}{|c|c|c|c|c|c|c|c|}
\hline \multirow[b]{2}{*}{ Subescalas SOCCS } & \multicolumn{2}{|c|}{$\begin{array}{c}\text { Femenino } \\
(\mathrm{n}=92)\end{array}$} & \multicolumn{2}{|c|}{$\begin{array}{l}\text { Masculino } \\
(\mathrm{n}=25)\end{array}$} & \multirow[b]{2}{*}{$\mathrm{t}$} & \multirow[b]{2}{*}{ gl } & \multirow[b]{2}{*}{ Sig. (2-tailed } \\
\hline & $M$ & $D E$ & $M$ & $D E$ & & & \\
\hline Actitudes & 5.75 & 1.26 & 5.73 & 1.33 & -.07 & 115 & .94 \\
\hline Destrezas & 3.67 & 1.37 & 3.97 & 1.22 & .97 & 115 & .33 \\
\hline Conocimientos & 3.87 & 1.13 & 4.35 & 1.10 & 1.85 & 115 & .06 \\
\hline Total & 4.45 & .80 & 4.68 & .78 & 1.30 & 115 & .19 \\
\hline
\end{tabular}

Nota: Elaboración propia.

Referente a la variable de escenario en el que laboran (sector público o privado), tampoco se encontró diferencia estadísticamente significativa en el promedio del SOCCS (promedio total, conocimientos, actitudes y destrezas). La puntuación total del personal consejero en rehabilitación para el sector público fue $(n=77, M=4.45, D E=.83)$, mientras para el sector privado fue $(n=21, M=$ $4.73, \mathrm{DE}=.69),(t(-1.39)=96, p=.16)$. En la Tabla 3 se detallan los resultados por subescala.

Tabla 3: Diferencia en las competencias de participantes en función del escenario en el que laboran (sector público o privado)

\begin{tabular}{lcccccccc}
\hline & \multicolumn{2}{c}{$\begin{array}{c}\text { Público } \\
(\mathrm{n}=77)\end{array}$} & \multicolumn{2}{c}{$\begin{array}{c}\text { Privado } \\
(\mathrm{n}=21)\end{array}$} & & & \\
\cline { 2 - 5 } Subescalas SOCCS & $\mathrm{M}$ & $\mathrm{DE}$ & $\mathrm{M}$ & $\mathrm{DE}$ & $\mathrm{t}$ & $\mathrm{gl}$ & Sig. (2-tailed) \\
\hline Actitudes & 5.57 & 1.35 & 6.09 & .83 & -1.664 & 96 & .09 \\
Destrezas & 3.78 & 1.35 & 3.83 & 1.31 & -16 & 96 & .87 \\
Conocimientos & 3.98 & 1.12 & 4.26 & 1.11 & -1.03 & 96 & .30 \\
\hline Total & 4.45 & .83 & 4.73 & .69 & -1.39 & 96 & .16 \\
\hline
\end{tabular}

Nota: Elaboración propia.

Al llevar a cabo un análisis de correlación de Spearman se encontraron relaciones negativas estadísticamente significativas entre años de experiencia de ejercicio de la profesión y la subescala de actitudes en el SOCCS $(r(-.252)=.006, p<.01)$. Ello implica que a más años de experiencia, menor nivel de actitudes competentes para la intervención con clientes LGB. También se encontró una relación negativa estadísticamente significativa entre los años de experiencia y la subescala de destrezas en el SOCCS $(r(-.239)=.010, p<.01)$. Esto implica que a más años de experiencia en el ejercicio de la profesión, menor nivel de destrezas competentes para la intervención con clientes LGB. En la Tabla 4 se desglosan los datos de correlación por cada subescala del SOCCS. 
doi: http://dx.doi.org/10.15359/ree.23-3.20

URL: http://www.una.ac.cr/educare

CORREO: educare@una.cr

Tabla 4: Relación entre los años de experiencia en ejercicio de la profesión de consejería en rehabilitación y las subescalas del SOCCS

\begin{tabular}{lcc}
\hline Competencias SOCCS & $r$ & $p$ \\
\hline Actitudes & $-.25^{* *}$ & .006 \\
Destrezas & $-.23^{* *}$ & .010 \\
Conocimientos & -.08 & .354 \\
\hline Total & -.02 & .828 \\
\hline
\end{tabular}

$\mathrm{P} \leq .05$

Nota: Elaboración propia.

\section{Discusión}

El personal consejero en rehabilitación debe prestar servicios competentes a diversas poblaciones (Departamento de Salud, 2013), incluidas las de orientación sexual LGB. El propósito de esta investigación fue explorar sus competencias para trabajar con las personas LGB. Los resultados reflejaron que el grupo participante presenta un nivel moderado de competencias, lo que sugiere que se perciben suficientemente capaces de trabajar con la población LGB. El valor medio total (4.49) de este personal se compara con los hallazgos de Henke et al. (2009) en un estudio realizado en consejerías de pareja y familia, que encontraron una puntuación total media en el SOCCS de 4.21 ( $\mathrm{DE}=0.80$ ). Mientras, en nuestro estudio, alcanzan una puntuación total media ligeramente superior a la del grupo consejero de pareja y familia en el estudio de Henke et al. (2009). Esto puede indicar que, como profesionales de la consejería en rehabilitación, al estar en una especialidad de la consejería cuyo propósito principal es proveer servicios a personas con diversidad funcional, se desarrollan competencias de consejería que pueden extrapolar con sus clientes LGB. Al respecto, una de las competencias que destacan Leahy, Chan y Saunders (2003), en las personas que practican la consejería en rehabilitación, es la defensa de sus clientes. Para esto, profesionales de la consejería en rehabilitación deben identificar y retar visiones estereotipadas, ayudar a afrontar diferentes situaciones psicosociales y educar a sus clientes sobre sus derechos en las leyes federales y estatales. Competencias que son necesarias para la provisión de servicios a clientes LGB, para favorecer que desarrollen poder y control sobre su identidad sexual y los asuntos psicosociales relacionados.

En cuanto a los resultados del grupo participante por subescala del SOCCS, obtuvo los puntajes más altos en el área de actitudes (5.79), seguida por el dominio de conocimientos (3.97) y por último el área de destrezas (3.72). El orden de mayor a menor competencias (actitudes, conocimientos y destrezas) en los resultados de las subescalas coincide con la tendencia emergente entre los estudios que han utilizado el SOCCS para medir las competencias del personal consejero para trabajar con los clientes LGB (por ejemplo, Bidell, 2005, 2012; Graham, 
Carney y Kluck, 2012; Henke et al., 2009). Presentar el resultado más alto en la subescala de actitudes podría asociarse a deseabilidad social, lo cual implica proyectar aceptación hacia la diversidad con base en lo socialmente esperado. Puede ser útil para futuras investigaciones estudiar la percepción de clientes LGB respecto a las actitudes que le muestran profesionales de la consejería en rehabilitación al proveer sus servicios.

De igual manera, presentar resultados moderados en la subescala de conocimiento implica que las personas que practican la consejería en rehabilitación deben continuar educándose con respecto a los problemas que afectan a sus clientes LGB. Deben comprender los modelos de desarrollo de identidad de LGB y estar familiarizados con otros modelos de desarrollo de identidad para grupos minoritarios para atender de manera competente a clientes LGB con múltiples identidades minoritarias (Israel et al., 2003).

Por otro lado, los sujetos participantes de esta investigación obtuvieron resultados moderados en el área de destrezas, pero fue el promedio más bajo, lo cual es cónsono con la investigación de Graham et al. (2012), quienes también utilizaron el SOCCS para conocer las competencias de personas que se capacitan para este campo. Presentar el promedio más bajos en el área de destrezas del SOCCS, sugiere que este personal consejero en rehabilitación siente que está moderadamente preparado por su formación y experiencia para trabajar con clientes de LGB. Esto evidencia la necesidad de proveerles la capacitación y experiencias de crecimiento profesional que resulten en mayores destrezas para la intervención con clientes LGB. Se ha demostrado que proveer servicios de educación continua y experiencias prácticas con la población LGB a profesionales de la consejería resulta en mayores niveles de destrezas y competencias generales en el SOCCS (Graham et al., 2012).

No se identificaron resultados estadísticamente significativos en cuanto a la relación entre competencias y las variables de género o el escenario laboral del grupo. Ello sugiere una percepción de competencias uniforme en términos del género y su escenario laboral.

Se encontró una relación negativa estadísticamente significativa entre los años de experiencia en ejercicio de la profesión de consejería en rehabilitación y las subescalas de destrezas y actitudes. Este hallazgo implica que a más años de experiencia, menores niveles de destrezas y actitudes, lo cual evidencia la necesidad de continuar reforzando la educación continuada que se provee al personal consejero en rehabilitación para que cuente con información actualizada y experiencias que le permitan perfeccionar sus destrezas de intervención. Además, es importante que estos grupos profesionales desarrollen buenas prácticas de consejería basadas en evidencia científica y que participen de actividades que les ayuden a manejar el estrés y evitar la quemazón laboral. Para esto es imprescindible que se provean procesos de supervisión continuos mediante los cuales se salvaguarde tanto el bienestar del grupo profesional como el de sus clientes (Cashwell y Dooley, 2001). 
doi: http://dx.doi.org/10.15359/ree.23-3.20

URL: http://www.una.ac.cr/educare

CORREO: educare@una.cr

\section{Implicaciones}

El nivel moderado de competencias evidenciado por los sujetos participantes en esta investigación implica que pueden continuar desarrollando mayores niveles de competencias para la intervención con clientes LGB. Para esto se requiere de variados esfuerzos que deben comenzar con la academia y las asociaciones profesionales, proveyendo cursos de educación continuada que aborden directamente estas temáticas.

En dichos cursos se debe promover la adquisición de conocimientos, destrezas y actitudes para trabajar con las situaciones que enfrentan la población LGB. Una vez que los grupos profesionales están en los escenarios de trabajo resulta esencial reforzar la supervisión clínica. Se entiende que la participación regular en procesos de supervisión clínica aumenta sus creencias de autoeficacia (Cashwell y Dooley, 2001). Existen cuatro tipos de experiencias que aumentan las creencias de autoeficacia: a) las experiencias que permiten dominar la tarea, b) las experiencias de aprendizaje vicario, c) la persuasión verbal, que incluye recibir retroalimentación sobre el progreso, y d) la excitación emocional (Bandura, 1977). A continuación, analizamos cómo, desde la supervisión clínica en la consejería en rehabilitación, podemos promover estas experiencias para aumentar las creencias de autoeficacia en profesionales que intervienen con clientes LGB.

Primeramente, para tener experiencias que permitan dominar la tarea, el personal consejero en rehabilitación puede trabajar directamente con clientes LGB o participar en juegos de roles que les ayuden a aumentar la autoeficacia al realizar intervenciones con esta población (Daniels y Larson, 2001). Las experiencias de aprendizaje vicario pueden tener lugar en procesos de supervisión grupal en las que participen otros compañeros o compañeras de trabajo que comuniquen cómo trabajan con esta población.

Estas experiencias pueden incluir el modelado de parte de personal supervisor y las discusiones de casos presentados en videos o con otros recursos que demuestren los procesos de intervención.

La persuasión verbal puede ser la herramienta más poderosa para mejorar la autoeficacia de la consejería en rehabilitación a través de la supervisión. La retroalimentación verbal proporciona orientación a profesionales al comunicar progreso, fortalezas y áreas por mejorar (Barnes, 2004). Muchos sujetos supervisados dependen del personal supervisor para una retroalimentación detallada sobre su desempeño en los procesos de consejería.

Por último, la excitación emocional se manifiesta a menudo como la ansiedad experimentada por el personal durante la sesión de consejería. Al considerar las competencias para proveer consejería a clientes LGB, puede haber factores relacionados con las actitudes hacia las personas LGB que aumentan significativamente la ansiedad de cada profesional (es decir, la homofobia) y su desempeño. Una barrera como esta podría ser abordada a través de prácticas y procesos de supervisión activos. 


\section{Limitaciones}

En esta investigación se utilizó una medida de auto-reporte para conocer la autopercepción de profesionales de la consejería en rehabilitación sobre sus competencias para trabajar con la población LGB. No obstante, la autopercepción de la competencia no refleja necesariamente la competencia real. Como con cualquier estudio, particularmente con un tema sensible, existe la posibilidad de que la participación haya sido sesgada hacia quienes presentaban interés en el tema. Los estudios futuros contribuirían al abordaje de la temática usando métodos de investigación diversos (cuantitativos, cualitativos o mixtos) que permitan obtener la retroalimentación de clientes LGB sobre la conducta de las personas que practican la consejería en rehabilitación. Estos estudios podrían brindar información, des de la perspectiva de la clientela, del conocimiento que estos grupos profesionales muestran de los asuntos relacionados con las personas LGB y las habilidades terapéuticas que poseen.

Por último, las investigaciones futuras deben centrarse en otros factores que contribuyen a las competencias de las personas que practican la consejería en rehabilitación, como el número de clientes que se atienden en los procesos de consejería que se identifican como LGB u otra dentro del espectro de la identidad sexual humana (por ejemplo: transgénero, transexual, intersexual, queer, asexual, otros).

Además, se podría estudiar el impacto de las experiencias de formación profesional en programas académicos de posgrado y más allá.

\section{Conclusión}

El objetivo de esta investigación fue explorar la percepción de profesionales que ejercen la consejería en rehabilitación en Puerto Rico sobre sus competencias para trabajar con la población LGB. Se encontró que los sujetos que participaron del estudio presentan un nivel de competencia general moderado para trabajar con las personas LGB. También se encontró un nivel de competencia moderada en las áreas específicas (subescalas) de actitudes, conocimientos y destrezas, en el orden de mayor a menor competencia que se presenta.

Por otro lado, se auscultó la relación entre las competencias y las variables de género, escenario laboral y años de experiencia en el ejercicio de la profesión. Al respecto, no se identificaron resultados estadísticamente significativos en torno a la relación entre competencias de participantes y variables de género y el escenario laboral de las consejerías en rehabilitación. No obstante, se encontró una relación negativa estadísticamente significativa entre los años de experiencia y las subescalas de destrezas y actitudes. Esto refuerza el argumento de que profesionales de la consejería en rehabilitación deben participar en programas de educación continua y experiencias prácticas que les permitan continuar aumentando sus niveles de competencias. 
doi: http://dx.doi.org/10.15359/ree.23-3.20

URL: http://www.una.ac.cr/educare

CORREO: educare@una.cr

El personal consejero en rehabilitación tiene la responsabilidad de servir a poblaciones diversas, sobre todo, aquellas que confrontan retos para lograr la plena inclusión en la sociedad. Promover la equidad y satisfacer las necesidades de las personas LGB también es un asunto que debe priorizar. Para lograr efectividad en las intervenciones con clientes LGB, las personas que ejercen la consejería en rehabilitación necesitan conocimiento de esta población, autoconocimiento de las actitudes personales hacia esta y aprender destrezas para ofrecerles consejería de manera competente. Con este propósito resulta imperioso que se autoevalúen continuamente las competencias para, de esta forma, desarrollarlas, reforzarlas o ayudar a corregirlas, y que se utilicen las actitudes, los conocimientos y las destrezas que mejor promuevan el bienestar de esta población. A su vez, podría facilitar que este personal consejero en rehabilitación interceda por sus clientes LGB, promoviendo su autodefensa, su apoderamiento y la justicia social.

\section{Referencias}

Arredondo, P., Toporek, R., Brown, S. P., Jones, J., Locke, D. C., Sanchez, J. y Stadler, H. (1996). Operationalization of the multicultural counseling competencies. Journal of Multicultural Counseling and Development, 24(1), 42-78. doi: https://doi.org/10.1002/j.2161-1912.1996. tb00288.x

Balkin, R. S., Schlosser, L. Z. y Levitt, D. H. (2009). Religious identity and cultural diversity: Exploring the relationships between religious identity, sexism, homophobia, and multicultural competence. Journal of Counseling \& Development, 87(4), 420-427. doi: https://doi. org/10.1002/j.1556-6678.2009.tb00126.x

Bandura, A. (1977). Self-efficacy: Toward a unifying theory of behavioral change. Psychological Review, 84(2), 191-215. Recuperado de https://www.uky.edu/ eushe2/Bandura/ Bandura1977PR.pdf

Barnes, K. L. (2004). Applying self-efficacy theory to counselor training and supervision: A comparison of two approaches. Counselor Education \& Supervision, 44(1), 56-69. doi: https://doi.org/10.1002/j.1556-6978.2004.tb01860.x

Bidell, M.P. (2005). The sexual orientation counselor competency scale: Assessing attitudes, skills, and knowledge of counselor working with lesbian, gay, and bisexual clients. Counselor Education and Supervision, 44(4), 267-279. doi: https://doi.org/10.1002/j.1556-6978.2005. tb01755.x

Bidell, M. P. (2012). Examining school counseling students' multicultural and sexual orientation competencies through a cross-specialization comparison. Journal of Counseling \& Development, 90(2), 200-207. doi: https://doi.org/10.1111/j.1556-6676.2012.00025.x 
Burckell, L. A. y Goldfried, M. R. (2006). Therapist qualities preferred by sexual-minority individuals. Psychotherapy: Theory, Research, Practice, Training, 43(1), 32-49. doi: http:// dx.doi.org/10.1037/0033-3204.43.1.32

Cashwell, T. H. y Dooley, K. (2001). The impact of supervision on counselor self-efficacy. The Clinical Supervisor, 20(1), 39-47. Retrieved from Education Research Complete database. doi: https://doi.org/10.1300/J001v20n01 03

Cochran, S. D., Sullivan, J. G. y Mays, V. M. (2003). Prevalence of mental disorders, psychological distress, and mental health services use among lesbian, gay, and bisexual adults in the United States. Journal of Consulting and Clinical Psychology, 71(1), 53-61. doi: http://dx.doi. org/10.1037/0022-006X.71.1.53

Daniel, J. H., Roysircar, G., Abeles, N. y Boyd, C. (2004). Individual and cultural-diversity competency: Focus on the therapist. Journal of Clinical Psychology, 60(7), 755-770. doi: https://doi.org/10.1002/jclp.20014

Daniels, J. A. y Larson, L. M. (2001). The impact of performance feedback on counseling selfefficacy and counselor anxiety. Counselor Education and Supervision, 41(2), 120-130. doi: http://dx.doi.org/10.1002/j.1556-6978.2001.tb01276.x

Departamento de Salud. (2013). Código de Ética de la Junta Examinadora de Profesionales de la Consejería en Rehabilitación de Puerto Rico. Puerto Rico: Junta Examinadora de Profesionales de la Consejería en Rehabilitación de Puerto Rico. Recuperado de https://www.scribd. com/document/208564220/Codigo-de-Etica-JECR-8442

Graham, S. R., Carney, J. S. y Kluck, A. S. (2012). Perceived competency in working with LGB clients: Where are we now? Counselor Education \& Supervision, 51(1), 2-16. doi: https://doi. org/10.1002/j.1556-6978.2012.00001.x

Green, E. J., McCollum, V. C. y Hays, D. G. (2008). Teaching advocacy counseling within a social justice framework: Implications for school counselors and educators. Journal for Social Action in Counseling and Psychology, 1(2), 14-30. Recuperado de https://openjournals.bsu. edu/jsacp/article/view/183/165

Henke, T., Carlson, T. S. y McGeorge, C. R. (2009). Homophobia and clinical competency: An exploration of couple and family therapists' beliefs. Journal of Couple \& Relationship Therapy, 8(4), 325-342. doi: https://doi.org/10.1080/15332690903246101

Herek, G. M.(2002). Heterosexuals'attitudes toward bisexual men and women in the United States. TheJournalofSexResearch,39(4),264-274.doi:https://doi.org/10.1080/00224490209552150 
doi: http://dx.doi.org/10.15359/ree.23-3.20

URL: http://www.una.ac.cr/educare

CORREO: educare@una.cr

Hernández, R., Fernández, C. y Beptista, P. (2010). Metodología de la investigación (6ª ed.). México: Mc Graw-Hill.

Israel, T., Ketz, K., Detrie, P. M., Burke, M. C. y Shulman, J. L. (2003). Identifying counselor competencies for working with lesbian, gay, and bisexual clients. Journal of Gay \& Lesbian Psychotherapy, 7(4), 3-21. doi: https://doi.org/10.1300/J236v07n04 02

Kocarek, C. y Pelling, N. (2003). Beyond knowledge and awareness: Enhancing counselor skills for work with gay, lesbian, and bisexual clients. Journal of Multicultural Counseling and Development, 31(2), 99-112. doi: https://doi.org/10.1002/j.2161-1912.2003.tb00536.x

Leahy, M. J., Chan, F. y Saunders, J. L. (2003). Job functions and knowledge requirement of certified rehabilitation counselors in the 21st century. Rehabilitation Counseling Bulletin, 46(2), 66-81. doi: https://doi.org/10.1177/00343552030460020101

Mays, V. M. y Cochran, S. D. (2001). Mental health correlates of perceived discrimination among lesbian, gay, and bisexual adults in the United States. American Journal of Public Health, 91(11), 1869-1876. doi: https://doi.org/10.2105/AJPH.91.11.1869

Meyer, I. H. (2003). Prejudice, social stress, and mental health in lesbian, gay, and bisexual populations: Conceptual issues in research evidence. Psychological Bulletin, 129(5), 674697. doi: https://doi.org/10.1037/0033-2909.129.5.674

Murphy, J. A., Rawlings, E. I. y Howe, S. R. (2002). A survey of clinical psychologists on treating lesbian, gay, and bisexual clients. Professional Psychology: Research and Practice, 33(2), 183189. doi: http://dx.doi.org/10.1037/0735-7028.33.2.183

Palma, T. V. y Stanley, J. L. (2002). Effective counseling with lesbian, gay, and bisexual clients. Journal of College Counseling, 5(1), 74-89. doi: https://doi.org/10.1002/j.2161-1882.2002. tb00208.x

Pearson, Q. M. (2003). Breaking the silence in the counselor education classroom: A training seminar on counseling sexual minority clients. Journal of Counseling and Development, 81(3), 292-300. doi: https://doi.org/10.1002/j.1556-6678.2003.tb00256.x

Rainey, S. y Trusty, J. (2007). Attitudes of master's-level counseling students toward gay men and lesbians. Counseling and Values, 52(1), 12-24. doi: https://doi.org/10.1002/j.2161007X.2007.tb00084.X

Richardson, T. Q. y Jacob, E. J. (2002). Contemporary issues in multicultural counseling: Training competent counselors. En J.Trusty, E. J. Looby y D. S. Sandhu (Eds.), Multicultural counseling. Context, theory and practice, and competence (pp. 31-53). Huntington, NY: Nova Publishers. 
Roe, R. A. (2002). What makes a competent psychologist? European Psychologist, 7(3), 192-202. doi: https://doi.org/10.1027//1016-9040.7.3.192

Satcher, J. y Schumacker, R. (2009). Predictors of modern homonegativity among professional counselors. Journal of LGBT Issues in Counseling, 3(1), 21-36. doi: https://doi. org/10.1080/15538600902754452

Sue, D. W., Arredondo, P. y McDavis, R. J. (1992). Multicultural counseling competencies and standards: A call to the profession. Journal of Counseling \& Development, 70(4), 477-486. doi: https://doi.org/10.1002/j.1556-6676.1992.tb01642.x

Villafañe, Á. A., Velázquez, J. L. y Báez, M. (2013). Evolución de la consejería en rehabilitación en Puerto Rico: Esfuerzos organizacionales y proyectos legislativos. Revista Electrónica Educare, 17(3), 117-133. Recuperado de http://www.scielo.sa.cr/pdf/ree/v17n3/a06v17n3 